\title{
Value-based routing of delayed intentions into brain-based vs external memory stores
}

Dawa Dupont ${ }^{1,2}$, Qianmeng Zhu ${ }^{1}$, and Sam J. Gilbert ${ }^{1}$

1. Institute of Cognitive Neuroscience, University College London, UK

2. Department of Psychology, University of Copenhagen, Denmark

Keywords: Cognitive offloading, prospective memory, value, reminders, reward

Acknowledgements: Supported by ESRG grant ES/N018621/1. DD was supported by a grant from the Independent Research Fund Denmark (9037-00169B). Experiment 1 can be viewed at "http://samgilbert.net/demos/ValueOffloadingl". Experiment 2 can be viewed at "http://samgilbert.net/demos/ValueOffloading2" and was pre-registered at "https://osf.io/bvt4r/" (Zhu \& Gilbert, 2019). Experiment 3 can be viewed at "http://samgilbert.net/demos/ValueOffloading3" and was preregistered at "https://osf.io/te5wy/" (Dupont \& Gilbert, 2022). De-identified data for all three experiments along with code to reproduce the analyses and graphs can be found at "https://osf.io/vx2qp/" (Gilbert, 2022). An earlier version of this manuscript is available at "https://psyarxiv.com/wq7rs/" and some of the data were presented at the 2020 Annual Meeting of the Psychonomic Society.

\section{Address correspondence to:}

Sam Gilbert

Institute of Cognitive Neuroscience

17 Queen Square

London WC1N 3AZ

$\mathrm{UK}$

Email: sam.gilbert@ucl.ac.uk

Tel: +44(0)207679 1121

Fax: $+44(0) 2078132835$ 
Individuals have the option of remembering delayed intentions by storing them in internal memory or offloading them to an external store such as a diary or smartphone alert. How do we route intentions to the appropriate store, and what are the consequences of this? We report three experiments (two pre-registered) investigating the role of value. In Experiment 1, participants preferentially offloaded high-value intentions to the external environment. This improved memory for both high- and low-value content. Experiment 2 replicated the low-value memory enhancement even when only high-value intentions were offloaded. This provides evidence for a cognitive spillover effect: when high-value content is offloaded, internal memory becomes reallocated to low-value content instead. Experiment 3 confirmed a theoretical prediction of this account: participants had superior memory for low- than high-value content when the external store was removed. These results imply that value-based offloading can lead to a cognitive spillover effect from high- to low-value content, similar to the automatic allocation of 'spare' capacity that has been proposed in the domain of visual attention. Individuals prioritize high-value information for external memory; consequently, they can be left with predimonantly low-value information if it fails. 
Our ability to remember delayed intentions, sometimes known as prospective memory (Brandimonte et al., 1996; A.-L. Cohen \& Hicks, 2017; Kliegel et al., 2008;

Kvavilashvili, 1987; Rummel \& McDaniel, 2019; Scullin et al., 2015), is essential for everyday functioning. One may, for example, need to remember to buy groceries on the way home from work, go to an appointment next week, or take medication every night before going to bed. However, our unaided mental abilities are limited. Prospective memory is particularly unreliable, with failures of this form of memory estimated to comprise 50-70\% of everyday memory failures (Crovitz \& Daniel, 1984; Terry, 1988). To increase the chance of remembering delayed intentions, we can create external cues to aid our memory. This is known as intention offloading (Gilbert et al., 2022; Risko \& Gilbert, 2016). Setting alarms, writing notes, and leaving items in eye-catching locations are all examples of intention offloading, alleviating the cognitive demands associated with remembering intentions internally.

Recent studies have shown that external reminders improve memory for intentions (Gilbert, 2015a, 2015b; Gilbert et al., 2020; Jones et al., 2021). A multitude of factors influence individuals' decisions whether to offload intentions or remember them internally (see Gilbert et al., 2022 for a review). For example, people are more likely to offload intentions when they have a higher memory load or encounter interruptions (Gilbert, 2015a). Independently of this, people base their offloading decisions on a metacognitive evaluation of their memory ability (Boldt \& Gilbert, 2019; Gilbert, 2015b; Gilbert et al., 2020). That is, people are more likely to offload intentions when they believe they have a poor memory, regardless of objective memory ability (see also Dunn \& Risko, 2016; Hu et al., 2019). Additional factors that influence intention offloading include a preference to minimise the cognitive effort associated with remembering internally (Sachdeva \& Gilbert, 2020) and a tendency to 
repeat whatever strategy - internal memory or external offloading - that one has used in the past (Scarampi \& Gilbert, 2020). However, the effect of intention value on offloading has not previously been investigated experimentally.

Some intentions have a higher value attached than others. That is, fulfilling them is associated with a greater reward, or forgetting with a greater punishment. For example, taking your medicine will probably be considered of higher value than buying groceries on the way home from work. Accordingly, intentions with higher value are more likely to be remembered and executed. In experimental research, value is often operationalized in terms of monetary rewards (Walter \& Meier, 2014). For instance, Cook et al. (2015) found that memory for delayed intentions is enhanced when those intentions have a higher financial reward attached.

Other research has investigated the relationship between value and retrospective memory (see Knowlton \& Castel, 2022 for a recent review). Studies consistently find that participants have superior retrospective memory for items associated with higher value, as well as stronger context-item associations (e.g., Adcock et al., 2006; Hennessee et al., 2018, 2019). Evidence suggests that at least two distinct mechanisms contribute to this effect. First, individuals support retrieval of high-value items by engaging in more effective encoding strategies such as deep semantic encoding (M. S. Cohen et al., 2017). Second, independent of any deliberate encoding strategies, automatic encoding of high-value items is enhanced. This effect may be subserved by dopaminergic projections to the hippocampus (Adcock et al., 2006; Shohamy \& Adcock, 2010). The same mechanism is thought to account for the enhancement of memory by states of high curiosity (Gruber \& Ranganath, 2019). Another mechanism that may contribute to value-based modulation of retrospective 
memory is selective neuronal replay and overnight consolidation for items encountered close to the time of a reward (Braun et al., 2018).

In sum, studies of prospective memory (Cook et al., 2015), episodic memory (Knowlton \& Castel, 2022), spatial memory (Schwartz et al., 2020; Siegel et al., 2021) and working memory (Atkinson et al., 2021; Braun et al., 2018; Robison \& Unsworth, 2017) all point to an enhancement of memory by reward. However, whereas the studies reviewed above all required participants to use unaided memory, in everyday life we additionally have the opportunity to partner with external tools and devices to help us remember. How does reward influence memory systems that extend beyond the brain? Participants tend to self-report greater use of external reminders for highervalue intentions (Meacham \& Singer, 1977; Penningroth \& Scott, 2013), however the link between value and intention offloading has not previously been studied experimentally (though see recent work by Park et al., 2022).

The present study therefore had two aims. The first was to investigate how the value attached to intentions influences participants' selection of internal versus external memory stores to remember those intentions. We measured this directly, rather than relying on participant self-report measures which are susceptible to bias (Meacham \& Singer, 1977; Penningroth \& Scott, 2013). The second aim was to investigate the consequences of this selection process for the internal representation of intentions. In particular, we investigated how the use of external reminders might affect internal memory for high- versus low-value intentions when an external store fails.

It has been proposed that cognitive offloading may lead to a redistribution of cognitive capacity to other tasks or processes (Risko et al., in press). We refer to this as 'cognitive spillover'. This theoretical account fits well with the finding that individuals 
engage in cognitive offloading as a means of reducing cognitive effort (Sachdeva \& Gilbert, 2020), seeing as cognitive effort has been proposed to signal an opportunity cost indicating that cognitive resources may be better directed elsewhere (Kurzban et al., 2013). Consistent with this, when individuals believe that an external store is available for one set of to-be-remembered items, this can lead to improved memory for another set of items (Storm \& Stone, 2014) or even enhanced performance of an unrelated cognitive task (Runge et al., 2019).

These findings indicate that beliefs about the availability of an external store for one memory task can influence performance in a separate task. However, in these studies participants did not have a choice whether or not to offload items: either all or none of the items in a list were offloaded according to the experimental condition. By manipulating the value of items in the present study, this allowed us to investigate the possibility of a selective offloading effect. This, in turn, allowed us to test the theoretical prediction of a cognitive spillover account, which implies that selective offloading of some items should lead to a spillover of cognitive resources to the other items that were not offloaded.

\section{Experiment 1}

We developed a task to explore value-based selection of intentions for internal and external memory stores, by adapting the intention offloading task used in previous studies (Gilbert, 2015b; Gilbert et al., 2020). The value of intentions was manipulated via monetary incentives in a 2 x 2 design crossing value (high / low) with offloading (permitted / not allowed). Based on previous research (Cook et al., 2015), we expected that memory would be better for high- than low-value intentions. However, we 
considered two possible predictions for how this value effect might be influenced by offloading. On the one hand participants might selectively set reminders for highvalue intentions, which might further improve high-value performance relative to lowvalue performance. This would amplify the value effect. Alternatively, participants might preferentially set reminders for low-value intentions because high-value intentions are already remembered well with internal memory. In this case, the value effect might be diminished when offloading is permitted. The main purpose of Experiment 1 was to evaluate these two predictions.

\section{Method}

Transparency and openness. We report below how we determined our sample size, all data exclusions (if any), all manipulations, and all measures in the study. Data were analysed using $\mathrm{R}$, version 3.6.2. All data and analysis code are available at "https://osf.io/vx2qp/". The full experiment can be viewed at "http://samgilbert.net/demos/ValueOffloadingl". This study's design and analysis were not pre-registered.

Participants. 27 volunteers took part in the experiment (15 female, 12 male; mean age: 24.4; range: 18-40). We based this sample size on previous data from our lab showing a within-subject effect of value on accuracy with effect size $d z=0.66$ (Aichelburg, 2018, p. 147). 27 participants gave $90 \%$ power to detect an effect of this magnitude in a paired t-test with alpha set to $.05(\mathrm{G} *$ Power version 3.1). All participants provided informed consent before participating and the research was approved by the UCL Research Ethics Committee (1584/002). 
Value-Intention Offloading Task. On each trial, 6 yellow circles numbered 1-6 were randomly positioned within a box on a touchscreen tablet device (Figure 1). The top and bottom sides of the box were black; the left and right sides were blue and pink, respectively. Participants were instructed to drag the circles in numerical sequence (1, 2,3 , etc.) to the bottom of the box, using their finger. Each time a circle was dragged to the bottom of the box a new circle appeared on the screen, occupying the position of the circle that had been removed. These new circles continued the numerical sequence (e.g., after dragging number 1 to the bottom, number 7 appeared in its place, and so on). Occasionally, the new circles appeared in blue or pink, corresponding with the left and right side of the box, respectively. Two seconds after appearing on the screen, their colour then faded to yellow so that they matched the other circles. A circle temporarily appearing in blue or pink represented an instruction that participants should form a delayed intention to drag that circle to its corresponding side of the box when it was reached in the sequence (e.g., if number 7 originally appeared in blue after dragging number 1 to the bottom, the participant should next drag numbers 2-6 to the bottom of the box, then drag number 7 to the blue side). Participants earned points when the blue and pink circles were dragged to the instructed side of the box. These points determined the payment at the end of the experiment. They received 10 points for one colour and 1 point for the other colour (counterbalanced between participants). If they incorrectly dragged a circle to the left/right of the box, they lost 1 point. Participants did not receive any points for circles dragged to the bottom. On each trial, there were 40 circles in total and 20 of them were either blue or pink. The number of points and a corresponding amount of money that the participants had earned so far was shown at the top of the screen, as 
well as a timer counting down from 2 minutes to encourage them to complete each trial quickly.

For half of the experimental trials ('offloading condition'), participants were given a free choice to set external reminders to help them remember the delayed intentions. They created reminders by immediately dragging target circles next to their instructed location (e.g., as soon as a blue number 7 appeared on the screen, the participant could drag this circle next to the blue side of the box). The initial placement of the circles at the beginning of each trial avoided the edges of the box. Therefore, when a circle was placed at one of the edges, this stood out and acted as a noticeable reminder for where it should eventually be dragged. An everyday analogy would be leaving an object that you will need tomorrow next to the front door, so that you remember to take it with you when you leave the house the following day. After setting a reminder, the screen faded to grey and the circles could not be dragged for 2.5 seconds, so there was a time cost associated with using this strategy. The 2.5 second duration for this 'lockout' period was chosen on the basis of pilot testing, in order to avoid ceiling effects in reminder-setting behaviour.

In the other half of the experimental trials ('nonoffloading condition'), participants were forced to rely on internal memory only. This was enforced by fixing the positions of all circles on screen apart from the next in the sequence, so that it was not possible to move forthcoming targets when they first appeared on the screen. Participants were asked to complete the task as quickly and accurately as possible.

[Figure 1 about here] 
Procedure. The task was performed on a Samsung SM-T580 Galaxy tablet computer, using the touchscreen interface. Participants were tested individually at the Institute of Cognitive Neuroscience, UCL. There were 16 experimental trials in total (each containing 10 high-value and 10 low-value targets). Participants performed one condition in the first half of the experiment and the other in the second half, counterbalanced between participants. At the end of the experiment, they received $£ 0.40$ for every 100 points they earned, plus an additional base payment of $\mathcal{E}^{3}$ for taking part, so the final payment was between $£ 3$ and $£ 10.04$ depending on performance.

Data analysis. Six measures were analysed: High-value target accuracy and Low-value target accuracy was the proportion of 10-point and 1-point targets, respectively, that were dragged to their instructed location. These were calculated separately for the offloading and nonoffloading conditions. High-value offloading proportion and Low-value offloading proportion was the proportion of high- and low-value targets, respectively, for which participants set up an external reminder, by moving it to a new location before reaching its position in the ongoing task. These were calculated for the offloading condition only. In common with our earlier work using related tasks (e.g., Gilbert, 2015a), the proportion of nontarget circles that were moved before their turn in the sequence was subtracted from these offloading proportions. This corrects for any tendency to accidentally drag the wrong circle, rather than deliberately setting a reminder. All tests were two-tailed.

\section{Results}


No participants met the planned exclusion criteria of performance more 2.5 standard deviations of the group mean for any of the dependent measures. See Figure 2 for a summary of results. The accuracy data were analysed in a 2 x 2 repeated-measures ANOVA with factors Offloading (offloading, nonoffloading) and Value (high, low). There were significant main effects of both Offloading, $F(1,26)=19.92, p<.001, \eta_{\mathrm{p}}{ }^{2}$ $=.43$ and Value, $F(1,26)=36.17, p<.001, \eta_{\mathrm{p}}{ }^{2}=.58$, reflecting higher accuracy in the offloading condition and for high-value targets. There was also a significant Offloading $\mathrm{x}$ Value interaction, $F(1,26)=6.78, p=.015, \eta_{\mathrm{p}}^{2}=.21$, indicating that the offloading condition increased accuracy for low-value targets more than high-value targets. Accuracy was significantly higher for high- than low-value targets in both the nonoffloading condition, $t(26)=5.75, p<.001, d z=1.11$, and the offloading condition, $t(26)=5.12, p<.001, d z=0.99$. Furthermore, accuracy was significantly higher in the offloading compared with the nonoffloading condition for both highvalue targets, $t(26)=3.40, p=.002, d z=0.65$, and low-value targets, $t(26)=4.09, p<$ $.001, d z=0.79$. In the offloading condition, the high-value offloading proportion was greater than the low-value offloading proportion, $t(26)=4.43, p<.001, d z=0.85$.

[Figure 2 about here]

\section{Discussion}

Replicating previous findings, we found that participants performed better for highthan low-value intentions when they used unaided memory (Cook et al., 2015). We also found that accuracy was increased when an offloading strategy was available (Gilbert, 2015a; Gilbert et al., 2020). Initially, we considered two hypotheses for the offloading condition: participants might preferentially offload high-value targets, 
amplifying the effect of value on accuracy, or they might preferentially offload lowvalue targets, diminishing any effect of value. Neither of these hypotheses was correct. We found that participants preferentially offloaded high-value targets, but the increase in accuracy was greatest for low-value targets.

How might this be explained? One possibility is a ceiling effect: participants already perform closer to $100 \%$ for high-value targets in the nonoffloading condition, so the potential improvement is greater for low-value targets even though participants set fewer reminders for these targets. An additional possibility is a cognitive spillover effect. Previous work in the field of cognitive offloading suggests that once participants offload memory to an external store, they dismiss the offloaded information from internal memory (Eskritt \& Ma, 2014; Kelly \& Risko, 2019a; Sparrow et al., 2011). This, in turn, may boost memory for subsequently-presented information (Storm \& Stone, 2014) or enhance performance of an unrelated task (Runge et al., 2019). Accordingly, it is possible that once participants offloaded high-value intentions, they reallocated memory resources to low-value intentions. This means that accuracy for low-value intentions could be increased not only due to external reminders, but also due to a spillover of internal memory resources to low-value information once highvalue information had been stored externally. Experiment 2 tested this hypothesis more directly.

\section{Experiment 2}

According to the cognitive spillover hypothesis, accuracy for low-value intentions is increased by high-value offloading, as a result of reallocated memory capacity. This implies that high-value offloading alone is sufficient to improve low-value accuracy; it 
should not be necessary to offload low-value intentions to observe such an effect. Experiment 2 tested this prediction by allowing participants to offload high-value intentions only, examining the influence of this on low-value accuracy.

\section{Method}

Transparency and openness. We report below how we determined our sample size, all data exclusions (if any), all manipulations, and all measures in the study. Data were analysed using $\mathrm{R}$, version 3.6.2. All data and analysis code are available at "https://osf.io/vx2qp/". The full experiment can be viewed at "http://samgilbert.net/demos/ValueOffloading2". This study's design and analysis was pre-registered at "https://osf.io/bvt4r/".

Participants. 27 participants took part in the experiment (19 female, 8 male; mean age: 23.4; range: 18-40). We performed a power calculation based on the difference in lowvalue accuracy between the offloading and nonoffloading condition in Experiment 1 $(d z=0.79)^{1}$. For $90 \%$ power to detect an effect of this magnitude, a sample size of 19 would be required. Seeing as this is smaller than the sample size in Experiment 1, we chose to test 27 participants, for consistency with the earlier experiment. This would give $>97 \%$ power to detect an effect of this magnitude $(G *$ Power 3.1$)$. All participants provided informed consent before participating, and the research was approved by the UCL Research Ethics Committee (1584/002).

\footnotetext{
${ }^{1}$ In the original pre-registration, this effect size was incorrectly reported, however this did not affect the overall conclusions of the power calculation.
} 
Procedure. Experiment 2 followed the same design as Experiment 1, except that in the offloading condition only high-value targets could be offloaded. The positions of lowvalue targets were fixed in the offloading condition in the same way as they were in the nonoffloading condition. In all other respects the experiments were identical.

Data analysis. All analyses were conducted as described in the preregistered plan. There were five key dependent measures. High-value target accuracy and low-value target accuracy were calculated separately for the offloading and nonoffloading conditions. High value offloading proportion was calculated for the offloading condition only. All tests were two-tailed.

\section{Results}

No participants met the pre-registered exclusion criteria. See Figure 3 for a summary of results. Data were analysed in a 2 x 2 repeated-measures ANOVA with factors Offloading (offloading, nonoffloading) and Value (high, low). This showed significant main effects of both Offloading, $F(1,26)=33.46, p<.001, \eta_{\mathrm{p}}^{2}=.56$, and Value, $F(1$, 26) $=39.87, p<.001, \eta_{\mathrm{p}}{ }^{2}=.61$, consistent with the results of Experiment 1 . The Offloading x Value interaction was not significant, $F(1,26)=0.25, p=.62, \eta_{\mathrm{p}}{ }^{2}=$ .009. Paired-sample t-tests showed that accuracy was significantly higher for highthan low-value targets in both the nonoffloading condition, $t(26)=6.78, p<.001, d z$ $=1.30$, and the offloading condition, $t(26)=5.10, p<.001, d z=0.98$. Furthermore, accuracy was significantly higher in the offloading than the nonoffloading condition for both high-value targets, $t(26)=6.56, p<.001, d z=1.26$, and - of particular importance for this experiment - for low-value targets, $t(26)=4.39, p<.001, d z=$ 
0.85. Therefore, accuracy for low-value targets was increased when it was possible to offload high-value targets.

[Figure 3 about here]

There was considerable variation in the proportion of high-value targets that participants offloaded when this was possible, spanning the full range from 0-1. Correlation analyses showed that proportion of high-value targets offloaded did not correlate significantly with the difference in high-value accuracy between the offloading and nonoffloading conditions, $r(25)=.17, p=.39$. However, the difference in low-value accuracy did correlate significantly with the proportion of high-value targets offloaded, $r(25)=.59, p<.001$. Therefore, accuracy for low-value targets increased in line with the proportion of high-value targets offloaded.

\section{Discussion}

Allowing participants to offload high-value targets improved memory for both highand low-value targets. This suggests that memory capacity was reallocated to lowvalue targets once it was no-longer occupied by high-value targets. The effect was underlined by a positive correlation between high-value offloading and the improvement in low-value accuracy. A similar association between high-value offloading and high-value accuracy may have been blunted by a ceiling effect due to the high baseline accuracy for high-value targets.

Although these results are consistent with the cognitive spillover account, they are not decisive. Another possible explanation is as follows: in the nonoffloading 
condition, participants need to make three-way discrimination for each item: 1)

nontarget, 2) low-value target, or 3) high-value target. Once participants set reminders for high-value targets, they are clearly cued. So for the remaining items, only a twoway discrimination is required: 1) nontarget, or 2) low-value target. It is possible that the improved accuracy for low-value targets in the offloading condition reflects the requirement for a simpler discrimination between the two response options for the nonoffloaded items, rather than three. The purpose of Experiment 3 was to further investigate the possibility of a cognitive spillover effect by probing internal memory directly.

\section{Experiment 3}

If participants reallocate memory resources to low-value content once those resources are no longer required for high-value content, we can make the following prediction based on the cognitive spillover account: once high-value content is offloaded, accuracy should be greater for low- than high-value content when internal memory is probed. In other words, without support from the external memory store, the typical memory enhancement for high- over low-value content (Cook et al., 2015) might not only be attenuated by offloading (as in Experiment 1), it may be reversed so that accuracy for low-value content should be greater than high-value content. We tested this prediction by adapting the task from Experiment 2 and occasionally probing internal memory.

\section{Method}


Transparency and openness. We report below how we determined our sample size, all data exclusions (if any), all manipulations, and all measures in the study. Data were analysed using R, version 3.6.2. All data and analysis code are available at "https://osf.io/vx2qp/". The full experiment can be viewed at "http://samgilbert.net/demos/ValueOffloading3". This study's design and analysis was pre-registered at "https://osf.io/te5wy/".

Participants. 104 participants took part in the study (40 female, 64 male, mean age: 34.39 , range 22-71 years). The sample size was based on the key prediction that once offloading is allowed, accuracy in a surprise memory test should be greater for lowthan high-value content. An initial pilot study $(n=27)$ indicated an effect size of $d z=$ 0.29 for this effect. In order to have $90 \%$ power to detect an effect of this magnitude with a one-tailed paired t-test and alpha set to 0.05 , a sample size of 104 was required (G*Power 3.1). Participants were recruited via the Amazon Mechanical Turk website (https://www.mturk.com). In order to reduce heterogeneity in performance, we restricted our sample to participants located in the USA with a $90 \%-100 \%$ approval rating.

Task. The task was based on Experiment 2 with six modifications. First, the number of points associated with high- versus low-value targets was changed from 10 versus 1 to 7 versus 3. This was based on initial pilot testing which suggested that Mechanical Turk participants were not sufficiently motivated to remember targets when they were only worth 1 point. Second, instead of 6 numbered circles on screen during the task, there were 12 circles. This increased the number of pending intentions that could be active at any one time, allowing a greater number of items to be tested when internal 
memory was probed. Third, each trial consisted of 36 circles to drag, of which 6 were low-value and 6 were high-value targets. This meant that each time a new circle appeared on the screen, there was a 50\% chance that it would be a target (i.e., 12 targets were randomly allocated to the 24 circles following the 12 initially presented on the screen). Fourth, there was no "lock-out" period as a result of reminder setting (i.e., the 2.5s inactivation period used in the earlier experiments). This was removed to maximise any difference in strategy between the offloading and nonoffloading conditions. Fifth, the timer at the top of the box counted down from 2 minutes and 30 seconds, rather than 2 minutes as in the earlier experiments. This gave participants more time to complete the task, because there were more circles on screen, increasing motor control demands, and participants would typically be using a computer mouse rather than a touchscreen to respond. The final modification was the addition of occasional surprise memory tests, in which the stimulus display was reset, and participants were asked to remember all pending targets using internal memory alone, without the benefit of any reminders they had set. Surprise test trials followed the design shown in Figure 4. These tests occurred at an unpredictable point between the $12^{\text {th }}$ and $24^{\text {th }}$ circle being dragged from the screen. The screen would go blank for $500 \mathrm{~ms}$, then the stimulus display would reappear with 11 circles arranged in random order vertically down the middle of the box. Three of these were low-value targets, three were high-value targets, and five were non-targets. Participants were asked to drag each of these 11 circles to the left, right, or bottom of the box as before. They were allowed to do this in any order, rather than having to follow the numerical sequence. A maximum of three circles could be dragged to the left and three to the right. 
[Figure 4 about here]

Procedure. Participants performed the task using their web browser on their home computer. There were a total of 16 trials (8 in each condition, in counterbalanced order). In the offloading condition, participants were able to set reminders for highvalue but not low-value targets. Surprise tests were presented on even-numbered trials. Therefore, each participant was tested on a total of 12 targets in each cell of the experimental design (4 surprise tests in each offloading condition, each of which contained 3 high-value and 3 low-value targets). Participants earned $\$ 1$ for every 100 points they scored in the main task, in addition to a base payment of $\$ 2$ for taking part, leading to a maximum possible payment of $\$ 9.60$. There was no additional payment associated with performance on the surprise memory tests.

Data analysis. All analyses were conducted as outlined in the preregistered plan, except where noted. There were nine dependent measures. Four measures were calculated separately for the offloading and nonoffloading conditions: High-value target accuracy, Low-value target accuracy, High-value surprise test accuracy, Low-value surprise test accuracy. Surprise test accuracy was calculated as the proportion of targets that were dragged to their instructed location rather than the bottom of the screen during surprise test trials. In addition, High-value target offloading proportion was calculated for the offloading condition. All tests were two-tailed unless otherwise specified.

\section{Results}

No participants met the pre-registered exclusion criteria. See Figure 5 for a summary of results. The target accuracy data from the trials that did not include a surprise test 
were analysed in a 2 x 2 repeated-measures ANOVA with factors Offloading (offloading, nonoffloading) and Value (high, low). This showed main effects of Offloading, $F(1,103)=41.36, p<.001, \eta_{\mathrm{p}}^{2}=.29$, and Value, $F(1,103)=112.92, p<$ $.001, \mathrm{n}_{\mathrm{p}}^{2}=.52$. As in Experiment 2, the Value $\mathrm{x}$ Offloading interaction was not significant, $F(1,103)=3.28, p=.07, \eta_{\mathrm{p}}^{2}=.03$. Paired sample t-tests showed that accuracy was significantly higher for high- than low-value targets in both the nonoffloading condition, $t(103)=8.29, p<.001, d z=0.81$, and the offloading condition, $t(103)=10.29, p<.001, d z=1.01$. Furthermore, accuracy was significantly higher in the offloading condition compared to the nonoffloading condition for both high-value targets, $t(103)=7.01, p<.001, d z=0.69$, and low-value targets, $t(103)=$ $3.61, p<.001, d z=0.35$.

There was considerable individual variation in the surprise test accuracy data (see Figure 5), potentially as a result of the small number of datapoints for each participant. These data were analysed in a 2 × 2 repeated-measures ANOVA with factors Offloading and Value. This showed a significant main effect of Value, $F(1$, $103)=7.54, p=.007, \mathrm{\eta}_{\mathrm{p}}^{2}=.07$, reflecting that surprise test accuracy on average was higher for high-value targets. The main effect of Offloading was not significant, $F(1$, $103)=3.84, p=.053, \eta_{\mathrm{p}}^{2}=.04$, but the Value $\mathrm{x}$ Offloading interaction was, $F(1,103)$ $=32.17, p<.0001, \mathrm{\eta}_{\mathrm{p}}{ }^{2}=.24$. This reflected the finding that permitting offloading reduced internal memory for high-value targets, $t(103)=4.85, p<.001$ (one-tailed),$d z$ $=0.48$, but increased internal memory for low-value targets, $t(103)=2.19, p=.015$ (one-tailed), $d z=0.21$. Furthermore, we found that surprise-test accuracy in the nonoffloading condition was greater for high- than low-value targets, $t(103)=6.96, p$ $<.001$ (one-tailed), $d z=0.68$. By contrast, in the offloading condition, surprise-test 
accuracy was significantly greater for low- than high-value targets, $t(103)=1.95, p=$ .027 (one-tailed), $d z=0.19$.

These results suggest that memory resources tend to be reallocated to lowvalue information once high-value information has been offloaded to an external store. However, there is an alternative possibility that could explain the enhanced memory for low-value items once high-value items have been offloaded ${ }^{2}$. According to this possibility, the act of offloading high-value items might act as a cue for participants to 'refresh' their internal memory for low-value items at the same time (cf. Sellen et al., 1997). We conducted an additional analysis to test this hypothesis (unlike the analyses above this was not included in our pre-registered plan). For the analysis of the offloading condition, we excluded all low-value items that were followed by any high-value offloading behaviour, before the surprise test. Therefore, there was no opportunity to refresh internal memory for these items. For the non-offloading condition, we applied the same restriction, based on the offloading behaviour from the equivalent trial of the offloading condition. This ensured that the targets compared between the two conditions were matched in terms of their position within a trial. The analysis showed that low-value items were better remembered in the offloading $(M=$ $0.412, S D=0.383)$ than the non-offloading condition $(M=0.355 ; S D=0.354 ; t(99)=$ $1.82, p=.036$ (one-tailed, as in the equivalent pre-registered analysis above), $d z=.15$ NB 4 participants were excluded from this analysis because they did not have any lowvalue items meeting our inclusion criteria in one or the other condition). Therefore, the offloading condition improved memory for low-value items, even when there was no subsequent offloading of high-value items, and hence no opportunity to refresh internal memory.

\footnotetext{
${ }^{2}$ We are grateful to an anonymous reviewer for suggesting this possibility.
} 


\section{Discussion}

As expected, in the nonoffloading condition, internal memory was greater for highthan low-value intentions. However, when offloading high-value intentions was permitted, this pattern was reversed: participants now had better memory for lowthan high-value targets. This suggests that internal memory is reallocated to low-value content once it is no longer occupied by high-value information.

The present results also help to clarify the effect of value on different types of memory task. The key factor that distinguishes a prospective from a retrospective memory task is that memory retrieval is self-initiated in a prospective memory task but more directly cued in a retrospective one (Craik, 1986; Einstein et al., 1995). In this way, the direct cue provided by the surprise memory tests in the present experiment can be considered to transform the prospective memory task into a retrospective memory task. Relatedly, the surprise memory tests probed what is sometimes termed (A.-L. Cohen et al., 2001) the 'retrospective component' of delayed intentions (remembering the detailed content of the intention) but not the 'prospective component' (remembering to interrupt ongoing behaviour at the appropriate moment, which allows the retrospective component can be executed). In the nonoffloading condition, memory was greater for high- than low-value intentions in both the main task (measuring prospective memory) and the surprise memory test (measuring retrospective memory). Therefore, results suggest that when they are not offloaded, high-value intentions are facilitated, at least in part, by enhancement of the retrospective content. By contrast, when they are offloaded, the retrospective content of intentions is weakened in internal memory. An interesting question for future 
research could be whether the prospective content is independently facilitated and weakened in an analogous way.

We conducted an additional analysis to investigate the possibility that offloading high-value items afforded an opportunity to refresh internal memory of low-value items. Results showed that memory for low-value items was improved, even when there was no opportunity for such a refresh process. Therefore, although it is possible that offloading high-value items could have cued participants to refresh internal memory for low-value items, this could not fully account for improved memory for low-value information when high-value information is offloaded.

\section{General Discussion}

An offloading strategy such as setting an external reminder carries both a cost the time and effort to set it up) and benefit (the increased chance of remembering, and the consequent reward). Seeing as it is not possible to set reminders for all of our intentions, we need to weigh these costs and benefits in order to select and prioritise information for offloading. Previous work shows that individuals do not always do this optimally (Gilbert et al., 2020, 2022). In this study, we asked two main questions. First, how does the value attached to an intention influence offloading? Second, what are the downstream consequences of this?

Value-based cognitive offloading

Experiment 1 showed that participants were much more likely to offload high- than low-value intentions. Therefore, value can be added to the factors that affect intention 
offloading, alongside additional factors established in previous work such as memory load and task interruption (Gilbert, 2015a), confidence (Gilbert, 2015b; Gilbert et al., 2020; Kirk et al., 2021), task instructions (Boldt \& Gilbert, 2019), effort-avoidance (Sachdeva \& Gilbert, 2020), previous reminder setting (Scarampi \& Gilbert, 2020), age (Scarampi \& Gilbert, 2021), and metacognitive interventions (Gilbert et al., 2020; though see also Engeler \& Gilbert, 2020; Grinschgl et al., 2020). The metacognitive model of cognitive offloading (Risko \& Gilbert, 2016), which considered the influence of just a single factor, should be updated to take account of these additional factors and their interactions.

As well as investigating the factors that influence the decision to offload, the present study also investigated the consequences of this. When individuals offload information to an external store, it subsequently becomes less accessible in internal memory (Henkel, 2014; Sparrow et al., 2011). Consistent with this, we found that internal memory for high-value intentions was reduced when they could be offloaded. This might be caused by a failure to apply encoding strategies such as subvocal rehearsal to offloaded information, and/or a process of intentional forgetting of offloaded info (Eskritt \& Ma, 2014; Kelly \& Risko, 2019a, 2019b). In this way, offloading an intention could act as a cue to forget, perhaps in much the same way that actually completing an intention can lead to it becoming deactivated and less accessible in memory (Bugg \& Scullin, 2013). This suggests that that the 'Zeigarnik' effect (Zeigarnik, 1938), whereby completed tasks are remembered less well than pending ones, might apply not only when a task is completed but also when it is offloaded.

A recent study by Park et al. (2022) also investigated the relationship between cognitive offloading, memory, and value. In Park et al.'s study, participants studied 
lists of words, each of which had an associated point value, and also typed the words into a computer. On two critical trials, participants believed that the typed words either would or would not be available at test. Park et al. found that participants had better memory for high- than low-value items when they did not expect to have access to the typed words and would therefore have to rely on their own memory. But when they expected to have access to the typed words, the effect of value was significantly reduced. Our results are consistent, seeing as we also found that the valueenhancement effect on memory was reduced when participants expected access to an external reminder. However, in our study this effect was not only attenuated, it was reversed: there was a low-value advantage.

There are two key differences between the present study and Park et al. (2022). First, participants in Park et al.'s experiments did not make any decisions whether or not to offload items, they simply typed every item into a computer. This means that Park et al. did not investigate whether value had any effect on the decision to offload, because participants always offloaded every item. Whereas Park et al.'s study investigated beliefs about whether an external store was available after all items were offloaded, the present study investigated the effect of voluntarily deciding to actually offload some items but not others. The second main difference between Park et al.'s study and the present work is that Park et al. did not include any additional memory task to probe whether offloading some items had any influence on memory for other items. Therefore Park et al.'s study did not investigate the cognitive spillover effect. 
Studies of directed forgetting have shown that instructing participants to forget one list of items can improve their memory for a subsequently-presented list (Bjork \& Bjork, 1996; Pastötter \& Bäuml, 2010; Sahakyan \& Kelley, 2002). Similarly, offloading prior information to an external store, can yield benefits for encoding of subsequently presented information (Storm \& Stone, 2014), or the performance of unrelated cognitive tasks (Runge et al., 2019). Consistent with this, we show here that offloading high-value intentions led to increased internal memory for low-value intentions. This provides evidence for a cognitive spillover effect: when high-value content is offloaded, internal memory becomes reallocated to low-value content instead. Unlike previous studies, where beliefs about the availability of an external store were manipulated but participants had no choice whether or not to offload (Runge et al., 2019; Storm \& Stone, 2014), the present study gave participants a voluntary choice whether or not to offload items. We found not only that the availability of an offloading strategy increased memory for non-offloaded items but also that individuals who actually offloaded more often showed a commensurate increase in memory for non-offloaded items.

In recent theoretical work by Risko and colleagues (in press), it has been suggested that two independent dimensions can be used to characterize the ways in which people use external memory stores. The substitutive-duplicative dimension characterizes whether people use an external store as a replacement for internal memory, or to duplicate items that are also stored in internal memory. The latter may occur particularly if the external store is unreliable. The redistribution dimension represents what people do with any processing capacity that is freed by cognitive offloading. According to Risko et al. (in press), individuals can either 'conserve' this capacity or redistribute it to other processing. 
However, an alternative account is possible, whereby cognitive resources freed by cognitive offloading automatically spill over into other cognitive activities without the option to conserve them. A model of this type has been proposed by Lavie (1995, 2005) in the domain of visual attention. According to perceptual load theory, visual attention resources are utilised by task-relevant features. Any remaining capacity then spills over to other visual features; it cannot be conserved. The present results are consistent with a similar model of cognitive spillover. Results from Experiment 2 showed a significant correlation between the amount of high-value offloading and the low-value memory enhancement. This suggests the automatic cognitive spillover from high- to low-value items, although of course it does not rule out the alternative possibility that this occurred as result of deliberate strategy. The automaticity of cognitive spillover could be tested in future studies by testing the boundary conditions and domain-specificity of spillover effects following cognitive offloading (cf. Runge et al., 2019). It cannot necessarily be assumed that following cognitive offloading, individuals have the option to choose between redistributing and conserving cognitive resources. Nor can it be assumed that cognitive resources automatically spill over. Arbitrating between these two positions (which perhaps lie on a spectrum rather than being dichotomous) will require further empirical work.

A link between cognitive offloading and cognitive spillover can also be made via the concept of cognitive effort (Inzlicht et al., 2018; Kool \& Botvinick, 2018; Kurzban et al., 2013). Recent work suggests that individuals engage in offloading, as least in part, as a means of reducing the cognitive effort that would otherwise be involved in a task (Sachdeva \& Gilbert, 2020). The function of cognitive effort, at least according to some theoretical accounts, is to signal an opportunity cost whereby cognitive processes could be better directed towards another activity (Kurzban et al., 
2013). This implies an intimate relationship between effort-minimisation and cognitive spillover. However, the extent to which spillover effects occur automatically or under voluntary control requires further empirical examination.

Value, prioritisation, and prospective memory

One consequence of the prioritisation of low-value items for internal memory once high-value items have been offloaded is that individuals can potentially be left with predominantly low-value information if an external store fails. This points to a potential cost of offloading to an unreliable external store: it can lead to low-value content being prioritised for brain-based cognitive resources, at the expense of highervalue content.

When external memory stores are available and reliable, especially with modern tools such as smartphones and wearable devices, the capacity of external memory is effectively unlimited. In this case, the key determinant for whether information is retained is not forgetting, it is whether or not that information was prioritised to begin with. Consistent with this, a naturalistic study of prospective memory by Marsh et al. (1998) showed that the main factor accounting for whether or not participants fulfilled their professed intentions over a 1-week period was not whether the intention was forgotten, but whether it was reprioritised. This raises the following question: how do individuals actually go about determining the value of everyday intentions, and thus prioritising them so that the most valuable ones can be offloaded?

The importance of intentions is determined by several factors including the reward attached, relative or absolute priority cues, and social motivation 
(Brandimonte et al., 2010; Brandimonte \& Ferrante, 2015; Penningroth \& Scott, 2007; Walter \& Meier, 2014). In the present study, the value of intentions was straightforward: high- and low-value intentions were matched in every respect apart from the financial reward attached. However, this is unlikely to be representative of everyday intentions, which are harder to compare on a single metric. For example, how exactly might intentions related to health, work, and leisure be compared and hence prioritised? Understanding the mechanisms by which these distinct value signals are integrated and compared could help explain how individuals select and prioritise intentions (Grünbaum et al., 2021).

Our results extend previous studies investigating value-based modulation of retrospective memory. Previous work has suggested that this modulation occurs as a result of two distinct mechanisms: selective engagement of effective encoding strategies for high-value items, along with automatic value-based potentiation of the encoding process (Knowlton \& Castel, 2022). These two mechanisms can be hard to distinguish when participants rely on brain-based memory alone. However, when an external reminder-setting strategy is available, this strategy can be directly observed. The results of Experiment 1 therefore provide additional support for the hypothesis that high-value items are preferentially remembered due to the selective engagement of memory strategies. Our results also diverge from previous studies of brain-based memory. In prior work, the distinct strategic and automatic encoding processes lead to the same outcome: in both cases the internal representation of high-value items is enhanced. By contrast, when an external offloading strategy is available - as it typically is in everyday life - these two mechanisms may have opposite effects. While automatic encoding of high-value items might still be enhanced, strategic encoding to an external memory store can diminish the internal representation of such items. 
In sum, we show here that participants offload intentions to an external store based on value, which can lead to internal memory being prioritised for lower-value information. Given that external memory aids such as smartphones and wearable devices can be highly reliable in providing time-, location-, and person-based reminders for delayed intentions once they are programmed to do so (Jones et al., 2021), this implies that understanding the valuation of intentions could be as important as understanding forgetting, if we are to understand the determinants of real-world prospective memory success. Future work should aim to investigate the mechanisms underlying this valuation process, its downstream effects on the accessibility of information in internal memory, and the relationship between cognitive offloading and cognitive spillover.

\section{Context}

This work arose from a programme of research seeking to contrast the factors that affect memory in the real world versus the laboratory. One factor that affects memory in the real world, but not usually the laboratory, is our tendency to use external tools and artefacts as part of the process of remembering. This has been investigated in recent research into cognitive offloading. Another factor that plays a key role in realworld memory, but not always in laboratory studies, is the influence of motivation and reward. We conducted these studies in an attempt to investigate what the relationship between these two factors might be. We have interpreted our results in terms of a reallocation of memory resources from high- to low-value information once highvalue information has been offloaded. However, we consider the concept of a memory 'resource' as a placeholder for further mechanistic explanation, rather than a full 
explanation in itself (Navon, 1984). Therefore, an aim for future studies is to reach a more detailed mechanistic understanding of value-based offloading and its consequences. 


\section{Figure captions}

Figure 1. Schematic illustration of the value-intention offloading task.

Figure 2. Results from Experiment 1. Red bars indicate standard errors corrected for the within-subject design using the method of Morey (2008). Error bars are therefore based on variability of within-subject effects regardless of between-subject variability in overall means, consistent with the inferential statistics presented in the text. Grey points and lines indicate data from individual participants, jittered to improve visualisation of overlapping points. $* * *$ indicates $\mathrm{p}<.001$ for the comparison between high- and low-value targets. Raw data and code to reproduce this figure can be downloaded at "https://osf.io/vx2qp/".

Figure 3. Results from Experiment 2. Left panel: accuracy data. Red bars indicate standard errors corrected for the within-subject design using the method of Morey (2008). Error bars are therefore based on variability of within-subject effects regardless of between-subject variability in overall means, consistent with the inferential statistics presented in the text. Grey points and lines indicate data from individual participants, jittered to improve visualisation of overlapping points. $* * *$ indicates $\mathrm{p}<.001$ for the comparison between high- and low-value targets. Right panel: scatter plot showing the proportion of high-value targets offloaded against the low-value accuracy improvement for each participant. Low-value accuracy improvement is the difference in low-value accuracy between the offloading and non-offloading conditions. Raw data and code to reproduce this figure can be downloaded at "https://osf.io/vx2qp/". 
Figure 4. Schematic illustration of the task in Experiment 3.

Figure 5. Results from Experiment 3. Red bars indicate standard errors corrected for the within-subject design using the method of Morey (2008). Error bars are therefore based on variability of within-subject effects regardless of between-subject variability in overall means, consistent with the inferential statistics presented in the text. Grey points and lines indicate data from individual participants, jittered to improve visualisation of overlapping points. *** indicates $\mathrm{p}<.001$ for the comparison between high- and low-value targets. * indicates $\mathrm{p}<.05$ one-tailed (as specified in the original pre-registration) for the comparison between high- and low-value targets. Raw data and code to reproduce this figure can be downloaded at "https://osf.io/vx2qp/". 
Figure 1

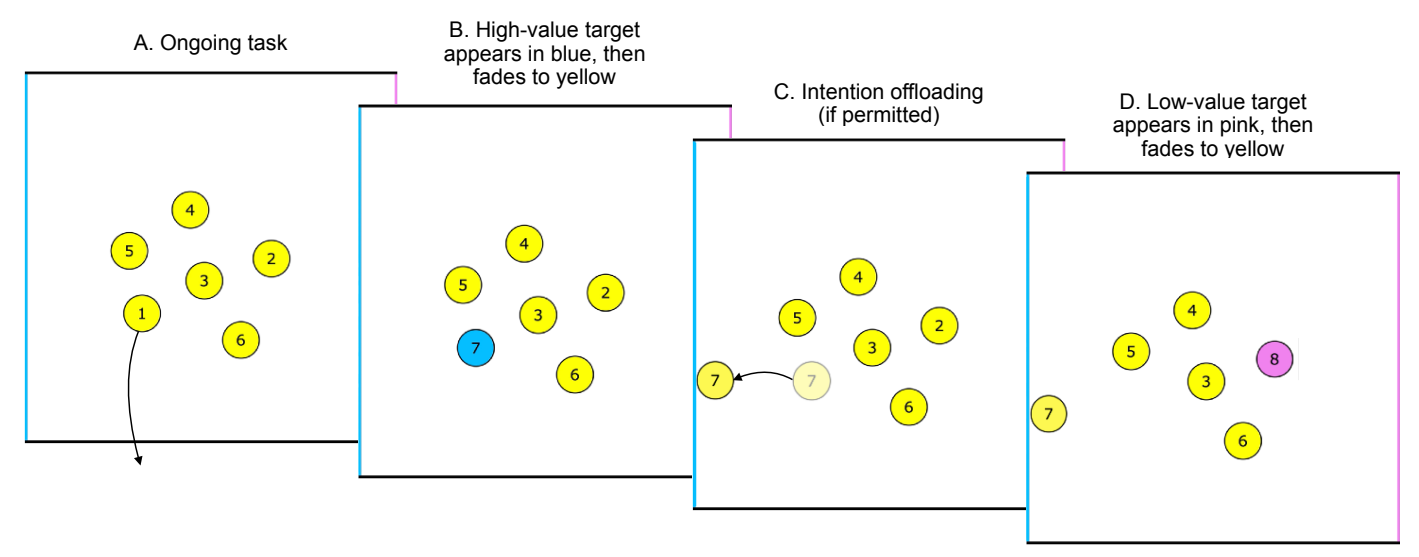

E. Intention offloading (if permitted)

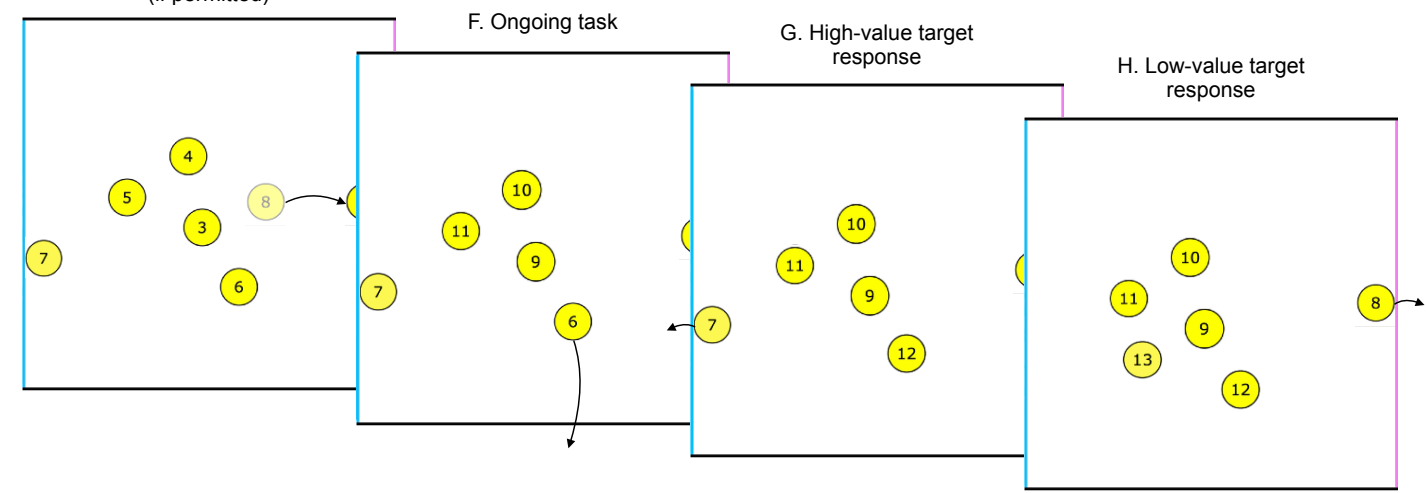


Figure 2
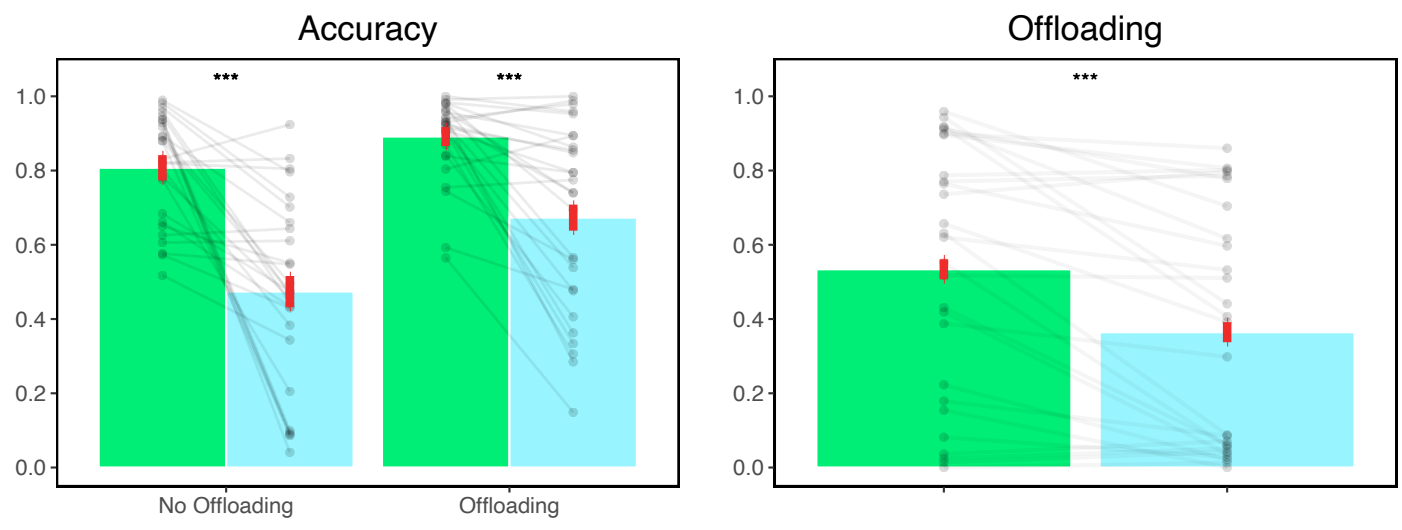

High-value targets

Low-value targets 
Figure 3
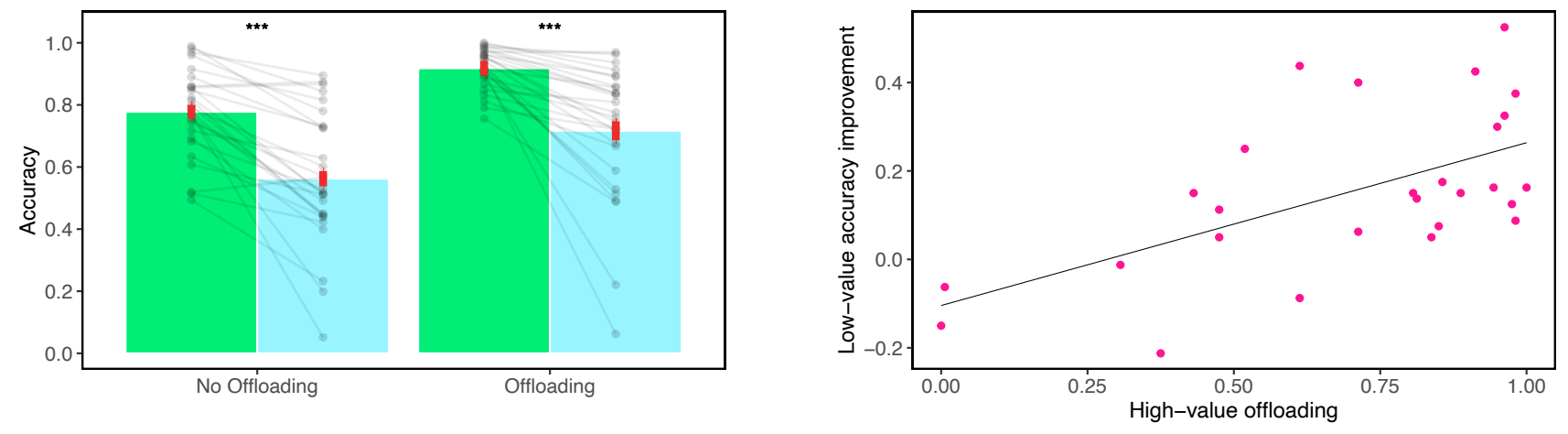

High-value targets o Low-value targets 
Figure 4

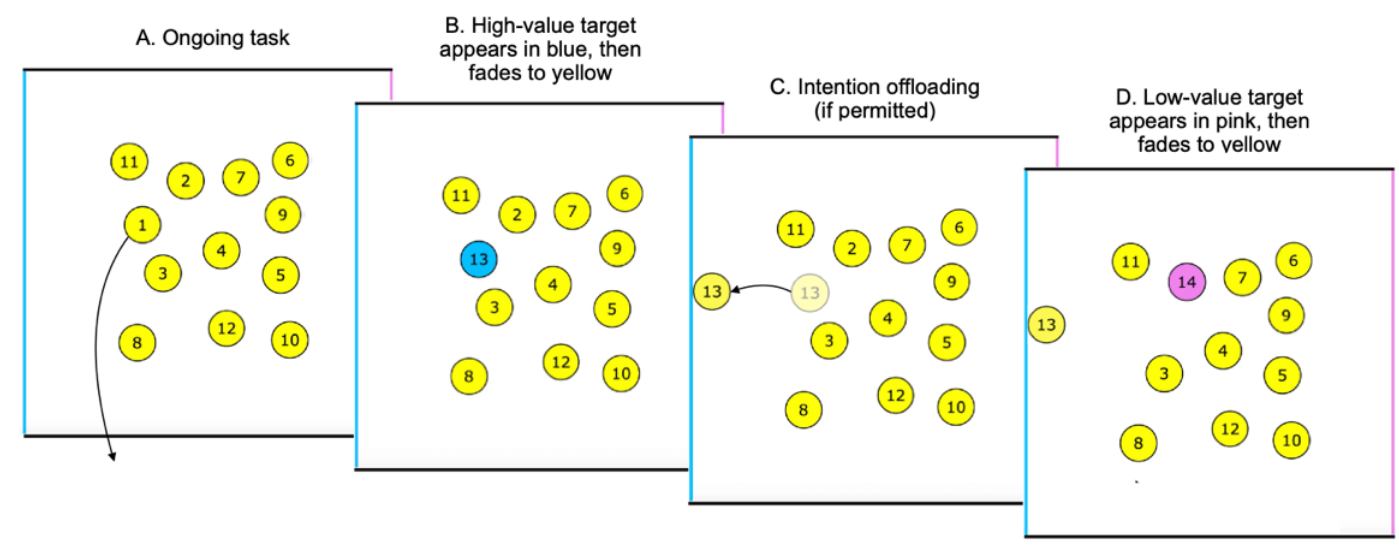

E. Ongoing task

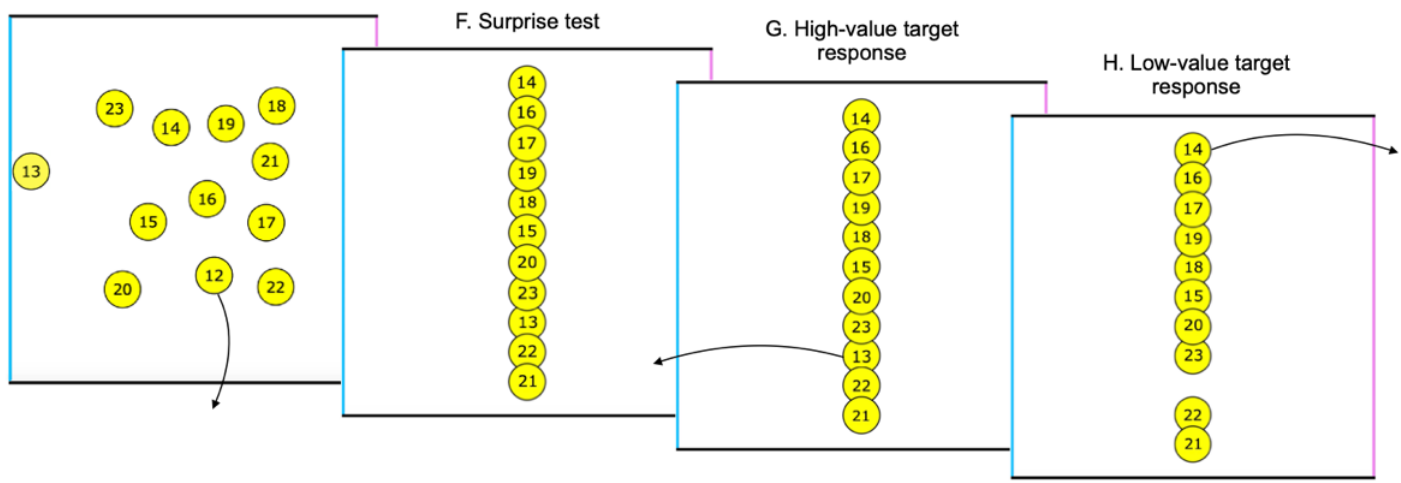


Figure 5
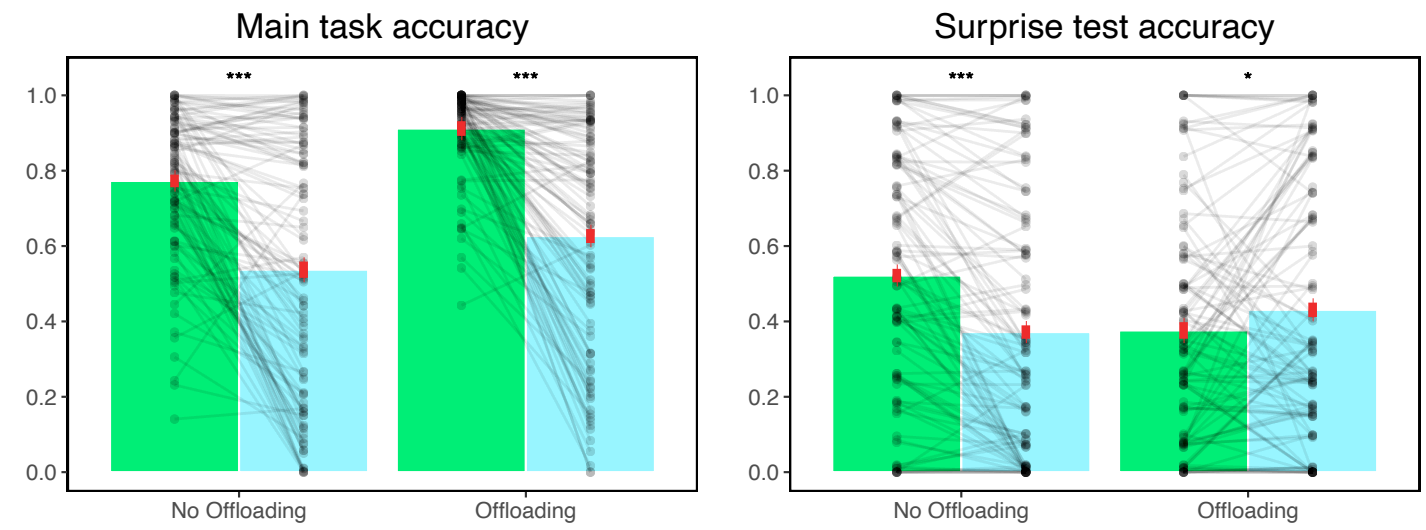

High-value targets Low-value targets 


\section{References}

Adcock, R. A., Thangavel, A., Whitfield-Gabrieli, S., Knutson, B., \& Gabrieli, J. D. E. (2006). Reward-Motivated Learning: Mesolimbic Activation Precedes Memory Formation. Neuron, 50(3), 507-517. https://doi.org/10.1016/j.neuron.2006.03.036

Atkinson, A. L., Allen, R. J., Baddeley, A. D., Hitch, G. J., \& Waterman, A. H. (2021). Can valuable information be prioritized in verbal working memory? Journal of Experimental Psychology: Learning, Memory, and Cognition, 47(5), 747-764. https://doi.org/10.1037/xlm0000979

Bjork, E. L., \& Bjork, R. A. (1996). Continuing Influences of To-Be-Forgotten Information. Consciousness and Cognition, 5(1-2), 176-196. https://doi.org/10.1006/ccog.1996.0011

Boldt, A., \& Gilbert, S. J. (2019). Confidence guides spontaneous cognitive offloading. Cognitive Research: Principles and Implications, 4(1), 45. https://doi.org/10.1186/s41235-019-0195-y

Brandimonte, M. A., Einstein, G. O., \& McDaniel, M. A. (1996). Prospective Memory: Theory and Applications. Psychology Press. http://www.amazon.co.uk/Prospective-Memory-Applications-MariaBrandimonte/dp/0805815368

Brandimonte, M. A., \& Ferrante, D. (2015). Effects of material and non-material rewards on remembering to do things Effects of material and non-material rewards on remembering to do things for others. 9(December). https://doi.org/10.3389/fnhum.2015.00647 
Brandimonte, M. A., Ferrante, D., Bianco, C., \& Grazia, M. (2010). Memory for prosocial intentions: When competing motives collide. Cognition, 114(3), 436-441. https://doi.org/10.1016/j.cognition.2009.10.011

Braun, E. K., Wimmer, G. E., \& Shohamy, D. (2018). Retroactive and graded prioritization of memory by reward. Nature Communications, 9(1), 4886. https://doi.org/10.1038/s41467-018-07280-0

Bugg, J. M., \& Scullin, M. K. (2013). Controlling Intentions: The Surprising Ease of Stopping After Going Relative to Stopping After Never Having Gone. Psychological Science. https://doi.org/10.1177/0956797613494850

Cohen, A., West, R., \& Craik, F. I. M. (2001). Modulation of the Prospective and Retrospective Components of Memory for Intentions in Younger and Older Adults. Aging, Neuropsychology, and Cognition, 8(1), 1-13.

Cohen, A.-L., \& Hicks, J. L. (2017). Prospective Memory: Remembering to Remember, Remembering to Forget (1st ed. 2017 edition). Springer.

Cohen, M. S., Rissman, J., Hovhannisyan, M., Castel, A. D., \& Knowlton, B. J. (2017). Free recall test experience potentiates strategy-driven effects of value on memory. Journal of Experimental Psychology: Learning, Memory, and Cognition, 43(10), 1581-1601. https://doi.org/10.1037/xlm0000395

Cook, G. I., Rummel, J., \& Dummel, S. (2015). Toward an understanding of motivational influences on prospective memory using value-added intentions. Frontiers in Human Neuroscience, 9(May), 1-15. https://doi.org/10.3389/fnhum.2015.00278

Craik, F. L. M. (1986). A functional account of age differences in memory: Mechanisms and performances. Human Memory and Cognitive Capabilities, August, 409-422. 
Crovitz, H. F., \& Daniel, W. F. (1984). Measurements of everyday memory: Toward the prevention of forgetting. Bulletin of the Psychonomic Society, 22(5), 413-414. https://doi.org/10.3758/BF03333861

Dunn, T. L., \& Risko, E. F. (2016). Toward a Metacognitive Account of Cognitive Offloading. Cognitive Science, 40(5), 1080-1127. https://doi.org/10.1111/cogs.12273

Dupont, D., \& Gilbert, S. J. (2022). The effect of setting external reminders for highvalue intentions on unaided memory for high-and low-value intentions: An online study. https://doi.org/10.17605/OSF.IO/TE5WY

Einstein, G. O., McDaniel, M. A., Richardson, S. L., Guynn, M. J., \& Cunfer, A. R. (1995). Aging and prospective memory: Examining the influences of selfinitiated retrieval processes. Journal of Experimental Psychology: Learning, Memory, and Cognition, 21(4), 996-1007. https://doi.org/10.1037/02787393.21 .4 .996

Engeler, N. C., \& Gilbert, S. J. (2020). The effect of metacognitive training on confidence and strategic reminder setting. PLOS ONE, 15(10), e0240858. https://doi.org/10.1371/journal.pone.0240858

Eskritt, M., \& Ma, S. (2014). Intentional forgetting: Note-taking as a naturalistic example. Memory \& Cognition, 42(2), 237-246. https://doi.org/10.3758/s13421-013-0362-1

Gilbert, S. J. (2015a). Strategic offloading of delayed intentions into the external environment. The Quarterly Journal of Experimental Psychology, 68(5), 971-992. https://doi.org/10.1080/17470218.2014.972963

Gilbert, S. J. (2015b). Strategic use of reminders: Influence of both domaingeneral and task-specific metacognitive confidence, independent of 
objective memory ability. CONSCIOUSNESS AND COGNITION, 33, 245-260. https://doi.org/10.1016/j.concog.2015.01.006

Gilbert, S. J. (2022). Value-based routing of delayed intentions into brain-based vs external memory stores. https://doi.org/10.17605/OSF.IO/VX2QP

Gilbert, S. J., Bird, A., Carpenter, J. M., Fleming, S. M., Sachdeva, C., \& Tsai, P.-C. (2020). Optimal use of reminders: Metacognition, effort, and cognitive offloading. Journal of Experimental Psychology: General, 149(3), 501-517. https://doi.org/10.1037/xge0000652

Gilbert, S. J., Boldt, A., Sachdeva, C., Scarampi, C., \& Tsai, P.-C. (2022). Outsourcing memory to external tools: A review of 'intention offloading' [Preprint]. PsyArXiv. https://doi.org/10.31234/osf.io/ahqtz

Grinschgl, S., Meyerhoff, H. S., Schwan, S., \& Papenmeier, F. (2020). From metacognitive beliefs to strategy selection: Does fake performance feedback influence cognitive offloading? Psychological Research. https://doi.org/10.1007/s00426-020-01435-9

Gruber, M. J., \& Ranganath, C. (2019). How Curiosity Enhances HippocampusDependent Memory: The Prediction, Appraisal, Curiosity, and Exploration (PACE) Framework. Trends in Cognitive Sciences, 23(12), 1014-1025. https://doi.org/10.1016/j.tics.2019.10.003

Grünbaum, B. T., Oren, F., \& Kyllingsbæk, S. (2021). A new cognitive model of long-term memory for intentions. Cognition, 215, 104817. https://doi.org/10.1016/j.cognition.2021.104817

Henkel, L. A. (2014). Point-and-Shoot Memories: The Influence of Taking Photos on Memory for a Museum Tour. Psychological Science, 25(2), 396-402. https://doi.org/10.1177/0956797613504438 
Hennessee, J. P., Knowlton, B. J., \& Castel, A. D. (2018). The effects of value on context-item associative memory in younger and older adults. Psychology and Aging, 33(1), 46-56. https://doi.org/10.1037/pag0000202

Hennessee, J. P., Patterson, T. K., Castel, A. D., \& Knowlton, B. J. (2019). Forget me not: Encoding processes in value-directed remembering. Journal of Memory and Language, 106, 29-39. https://doi.org/10.1016/j.jml.2019.02.001

Hu, X., Luo, L., \& Fleming, S. M. (2019). A role for metamemory in cognitive offloading. Cognition, 193, 104012. https://doi.org/10.1016/j.cognition.2019.104012

Inzlicht, M., Shenhav, A., \& Olivola, C. Y. (2018). The Effort Paradox: Effort Is Both Costly and Valued. Trends in Cognitive Sciences, 22(4), 337-349. https://doi.org/10.1016/j.tics.2018.01.007

Jones, W. E., Benge, J. F., \& Scullin, M. K. (2021). Preserving prospective memory in daily life: A systematic review and meta-analysis of mnemonic strategy, cognitive training, external memory aid, and combination interventions. Neuropsychology, 35(1), 123-140. https://doi.org/10.1037/neu0000704

Kelly, M. O., \& Risko, E. F. (2019a). Offloading memory: Serial position effects. Psychonomic Bulletin \& Review. https://doi.org/10.3758/s13423-01901615-8

Kelly, M. O., \& Risko, E. F. (2019b). The Isolation Effect When Offloading Memory. Journal of Applied Research in Memory and Cognition, S2211368119301585. https://doi.org/10.1016/j.jarmac.2019.10.001

Kirk, P. A., Robinson, O. J., \& Gilbert, S. J. (2021). Trait anxiety does not correlate with metacognitive confidence or reminder usage in a delayed intentions 
task. Quarterly Journal of Experimental Psychology, 74(4), 634-644. https://doi.org/10.1177/1747021820970156

Kliegel, M., McDaniel, M. A., \& Einstein, G. O. (2008). Prospective memory: Cognitive, neuroscience, developmental, and applied perspectives. In Prospective memory: Cognitive, neuroscience, developmental, and applied perspectives. Erlbaum. https://doi.org/10.4324/9780203809945

Knowlton, B. J., \& Castel, A. D. (2022). Memory and Reward-Based Learning: A Value-Directed Remembering Perspective. Annual Review of Psychology, 73(1), 25-52. https://doi.org/10.1146/annurev-psych-032921-050951

Kool, W., \& Botvinick, M. (2018). Mental labour. Nature Human Behaviour. https://doi.org/10.1038/s41562-018-0401-9

Kurzban, R., Duckworth, A., Kable, J. W., \& Myers, J. (2013). An opportunity cost model of subjective effort and task performance. The Behavioral and Brain Sciences, 36(6), 661-679. https://doi.org/10.1017/S0140525X12003196

Kvavilashvili, L. (1987). Remembering intention as a distinct form of memory. British Journal of Psychology, 78(April), 507-518. https://doi.org/10.1111/j.2044-8295.1987.tb02265.x

Lavie, N. (1995). Perceptual load as a necessary condition for selective attention. Journal of Experimental Psychology: Human Perception and Performance, 21(3), 451-468. https://doi.org/10.1037/0096-1523.21.3.451

Lavie, N. (2005). Distracted and confused?: Selective attention under load. Trends in Cognitive Sciences, 9(2), 75-82. https://doi.org/10.1016/j.tics.2004.12.004

Marsh, R. L., Hicks, J. L., \& Landau, J. D. (1998). An investigation of everyday prospective memory. Memory \& Cognition, 26(4), 633-643. 
Meacham, J. A., \& Singer, J. (1977). Incentive Effects in Prospective Remembering. The Journal of Psychology, 97(2), 191-197. https://doi.org/10.1080/00223980.1977.9923962

Morey, R. D. (2008). Confidence Intervals from Normalized Data: A correction to Cousineau (2005). Tutorials in Quantitative Methods for Psychology, 4(2), 61-64. https://doi.org/10.20982/tqmp.04.2.p061

Navon, D. (1984). Resources-A theoretical soup stone? Psychological Review, 91(2), 216-234. https://doi.org/10.1037//0033-295X.91.2.216

Park, J. S., Kelly, M. O., Hargis, M. B., \& Risko, E. F. (2022). The effect of external store reliance on actual and predicted value-directed remembering. Psychonomic Bulletin \& Review. https://doi.org/10.3758/s13423-02202064-6

Pastötter, B., \& Bäuml, K.-H. (2010). Amount of postcue encoding predicts amount of directed forgetting. Journal of Experimental Psychology: Learning, Memory, and Cognition, 36(1), 54-65. https://doi.org/10.1037/a0017406

Penningroth, S. L., \& Scott, W. D. (2007). A motivational-cognitive model of prospective memory: The influence of goal relevance. In Psychology of motivation (pp. 115-128). Nova Science Publishers.

Penningroth, S. L., \& Scott, W. D. (2013). Task importance effects on prospective memory strategy use. Applied Cognitive Psychology, 27(5), 655-662.

Risko, E. F., \& Gilbert, S. J. (2016). Cognitive Offloading. Trends in Cognitive Sciences, 20(9), 676-688. https://doi.org/10.1016/j.tics.2016.07.002

Risko, E. F., Kelly, M. O., Lu, X., \& Pereira, A. (in press). Varieties of Offloading Memory: A Framework. In Q. Wang \& A. Hoskins, The Remaking of 
Memory in the Age of Social Media and the Internet. Oxford University Press.

Robison, M. K., \& Unsworth, N. (2017). Working memory capacity, strategic allocation of study time, and value-directed remembering. Journal of Memory and Language, 93, 231-244.

https://doi.org/10.1016/j.jml.2016.10.007

Rummel, J., \& McDaniel, M. A. (Eds.). (2019). Prospective Memory (1st edition). Routledge.

Runge, Y., Frings, C., \& Tempel, T. (2019). Saving-enhanced performance: Saving items after study boosts performance in subsequent cognitively demanding tasks. Memory, 1-6. https://doi.org/10.1080/09658211.2019.1654520

Sachdeva, C., \& Gilbert, S. J. (2020). Excessive use of reminders: Metacognition and effort-minimisation in cognitive offloading. Consciousness and Cognition, 85, 103024. https://doi.org/10.1016/j.concog.2020.103024

Sahakyan, L., \& Kelley, C. M. (2002). A contextual change account of the directed forgetting effect. Journal of Experimental Psychology: Learning, Memory, and Cognition, 28(6), 1064-1072. https://doi.org/10.1037/02787393.28.6.1064

Scarampi, C., \& Gilbert, S. J. (2020). The effect of recent reminder setting on subsequent strategy and performance in a prospective memory task. Memory, 28(5), 677-691.

https://doi.org/10.1080/09658211.2020.1764974 
Scarampi, C., \& Gilbert, S. J. (2021). Age differences in strategic reminder setting and the compensatory role of metacognition. Psychology and Aging, 36(2), 172-185. https://doi.org/10.1037/pag0000590

Schwartz, S. T., Siegel, A. L. M., \& Castel, A. D. (2020). Strategic encoding and enhanced memory for positive value-location associations. Memory \& Cognition, 48(6), 1015-1031. https://doi.org/10.3758/s13421-02001034-4

Scullin, M. K., Mullet, H. G., Einstein, G. O., \& McDaniel, M. A. (2015). Prospective Memory. In International Encyclopedia of the Social \& Behavioral Sciences (pp. 270-278). Elsevier. https://doi.org/10.1016/B978-0-08-097086-

\section{$8.51055-1$}

Sellen, A. J., Louie, G., Harris, J. E., \& Wilkins, A. J. (1997). What Brings Intentions to Mind? An In Situ Study of Prospective Memory. Memory, 5(4), 483-507. https://doi.org/10.1080/741941433

Shohamy, D., \& Adcock, R. A. (2010). Dopamine and adaptive memory. Trends in Cognitive Sciences, 14(10), 464-472. https://doi.org/10.1016/j.tics.2010.08.002

Siegel, A. L. M., Schwartz, S. T., \& Castel, A. D. (2021). Selective memory disrupted in intra-modal dual-task encoding conditions. Memory \& Cognition, 49(7), 1453-1472. https://doi.org/10.3758/s13421-021-01166-1

Sparrow, B., Liu, J., \& Wegner, D. M. (2011). Google effects on memory: Cognitive consequences of having information at our fingertips. Science (New York, N.Y.), 333(6043), 776-778. https://doi.org/10.1126/science.1207745 
Storm, B. C., \& Stone, S. M. (2014). Saving-Enhanced Memory: The Benefits of Saving on the Learning and Remembering of New Information. Psychological Science. https://doi.org/10.1177/0956797614559285

Terry, W. S. (1988). Everyday Forgetting: Data from a Diary Study. Psychological Reports, 62(1), 299-303. https://doi.org/10.2466/pr0.1988.62.1.299

Walter, S., \& Meier, B. (2014). How important is importance for prospective memory? A review. Frontiers in Psychology, 5. https://doi.org/10.3389/fpsyg.2014.00657

Zeigarnik, B. (1938). On finished and unfinished tasks. In W. D. Ellis, $A$ source book of Gestalt psychology. (pp. 300-314). London, England: Harcourt, Brace and Co.

Zhu, Q., \& Gilbert, S. J. (2019). The effect of setting external reminders for highvalue intentions on unaided memory for low-value intentions. https://doi.org/10.17605/OSF.IO/D5BYH 